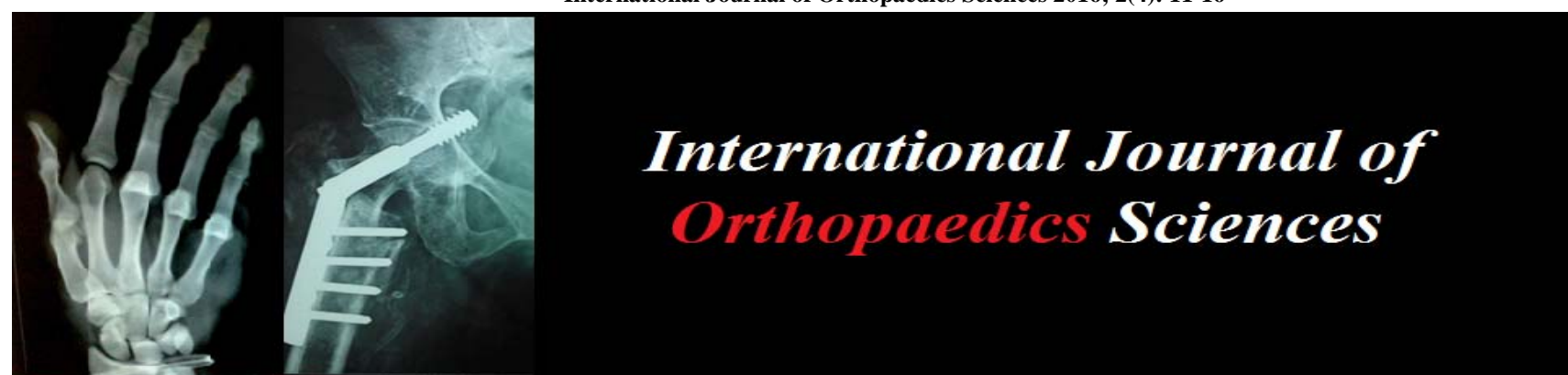

ISSN: 2395-1958

IJOS 2016; 2(4): 11-16

(C) 2016 IJOS

www.orthopaper.com

Received: 03-08-2016

Accepted: 04-09-2016

Dr. Sethu S

Assistant professor

Orthopaedics Govt. TDMC

Alapuzha Kerala, India.

Dr. Muhammed Sameeh

Assistant Professor

Govt. TDMC Alapuzha Kerala

Dr. Jithuram Jayaram

Senior Resident, Govt. TDMC

Alapuzha Kerala, India.

Dr. Sukesh AN

Junior Resident, Govt. TDMC

Alapuzha Kerala, India.
Correspondence

Dr. Sethu S

Assistant professor

Orthopaedics Govt. TDMC

Alapuzha Kerala, India.

\section{Stellate block using blunted needle as an OPD procedure in the symptomatic management of cervicobrachial neuralgia}

\author{
Dr. Sethu S, Dr. Muhammed Sameeh, Dr. Jithuram Jayaram and \\ Dr. Sukesh AN
}

DOI: $\underline{\text { http://dx.doi.org/10.22271/ortho.2016.v2.i4.003 }}$

\section{Abstract}

Background: Cervicobrachial neuralgia is a very common symptom of patients attending orthopaedics OPD. There are multitude of causes and some of them are life threatening. These patients are usually treated by Tricyclic ante depressants and similar drugs. Stellate ganglion block is a proven technique for the management of sympathetic mediated pain as is the case with most of these cervicobrachial neuralgias

Materials \& Methods: The study was done in Govt. TD Medical College, Alleppey, Kerala, India between a period of 2015 July and 2016 august for Adults with cervicobrachial neuralgia both acute and chronic evaluated clinically and radiologically to rule out malignancies and mechanical severe root compression. Total number of patients included in the study were 35 of which 10 were males and 25 were females. Three sessions of stellate block is performed in patients at biweekly intervals using $10 \mathrm{ml}$ $1 \%$ lignocaine with adrenaline. They were followed up at 1month 3 months 6 months and 1 year using VISUAL ANALOUGE SCALE.

Results: All petients were followed up for 1 year after 3 sessions of biweekly stellate block using blunted needle at 1 month 3 months 6 months and 1 year intervals. The outcomes were measured using Visual Analouge scale. At I year follow up 32 out of 35 patients had good results (VAS 0). 3 patients had fair results (VAS 1)

Conclusions: Among the options for the management of Cervicobrachial neuralgia stellate ganglion block is very effective and well documented method. When compared to stallate block, medical management using tricyclic antidepressants and similar drugs cause significant adverse reactions at therapeutic dosage. Stellate block using a blunted needle is a very safe method and nullifies the complications

Keywords: Stellate block; visual analogue scale, blunted needle

\section{Introduction}

Cervicobrachial neuralgia is a terminology used to describe the condition of vague but incapacitating pain involving the neck and adjoining upper limb and is most possibly sympathetically mediated. In simple terms, it is a neuralgia in which pain extends from the cervical region to the arms or fingers.

The incidence of cervicobrachial neuralgia is fairly common ${ }^{[1]}$ and at an average the attendance at our OPD is $10 \%$. Most of these patients voluntarily attend orthopaedics OPD as they themselves recognize the entity as something related to bone.

Any painful condition of cervical spine and adjoining area can lead to the symptomatology of cervicobrachial neuralgia, and the physician's task is to identify the underlying cause.

It is seen that even though the primary cause is identified and treated, the symptomatic relief that patient seeks demands prolonged use of analgesics, antidepressants and physical measures. If we are able to reduce the duration of symptoms and thereby the temporary disability, it will be highly ergonomical, as this condition mostly affects breadwinners of the community.

Evaluation of the role of stellate ganglion block as a day case procedure for the symptomatic management of cervicobrachial neuralgia is worth considering in the aforementioned circumstances. 


\section{Objectives}

1. To study the effectiveness of Stellate Ganglion Block using blunted needle in the symptomatic management of Cervicobrachial neuralgia as measured by Visual Analogue Scale ${ }^{[12]}$

2. To popularize this method among orthopaedic surgeons

\section{Review of literature}

The costs of unrelieved, chronic pain continue to be a major socioeconomic problem. Chronic, unrelieved pain is not only a major drain on scarce healthcare resources, but it is the cause of needless suffering of millions of people worldwide.

The basic task is to identify the cause of cervicobrachial neuralgia. By clinical, radiological and other evaluations it is never too difficult, to arrive at a diagnosis. Then it requires to be classified into 1 . Pain of terminal illness 2 . The pain of Non terminal illness.

Both types of cervicobrachial neuralgia require multidisciplinary measures. The first group is better managed by interventional pain therapist, who is a qualified anaesthesiologist. The second group, which forms the bulk, can be managed by Orthopaedic surgeons who are very familiar with the local Anatomy in their day to day practice. Theoretically it is possible to produce these symptoms by irritating the nerves (1) within the skull, (2) where the skull joins the spine, (3) within the cervical spinal column, (4) in the intervertebral foramina, (5) in the supraclavicular region, (6) in the shoulder, and (7) in the thorax.

\section{Usual causes of cervicobrachial neuralgia}

Cervicobrachial neuralgia (CBN) is frequent in outpatient clinic of in general medicine orthopaedics and rheumatology. Even though cervical disc degeneration and osteophytosis constitute the most frequent etiology of such a trouble, the practitioner must be aware of much more serious underlying cause.

\section{Cervical Spondylosis}

Cervical spondylosis is very common and is the cause of symptoms in a large number of people over the age of 45 . Kellgreen and Lawrence, as quoted by Lord Brian found radiological evidence of disc degeneration in over $80 \%$ of persons after the age of 55 in a large series of cases investigated.

Some of the symptoms of cervical spondylosis can be explained on an anatomical basis.

a) Paracentral osteophytes, arising from the Luschka and zygapophyseal (paravertebral) joints, encroach on the intervertebral foramen and give rise to pain in the neck and brachial radiculitis. If compression of the nerve root is severe, then wasting and weakness of the muscles of the arm and hand, associated with paraesthesia and reflex changes, will occur.

b) Centrally placed posterior osteophytes or bars. These can interfere with cord function, with the production of spastic paraparesis.

c) Degenerative changes in the intervertebral disc. The nucleus pulposus may either be absorbed and desiccated, or herniated backwards, where it can cause symptoms by direct pressure on the cord or nerve roots. When there is marked loss of disc height the bony rims of adjacent vertebral bodies actually come into direct contact with each other, and can pinch the annulus fibrosis, which is exceedingly pain-sensitive and contains sympathetic nerve endings supplied by the sinu-vertebral nerve. This direct weight-bearing and friction between the vertebral rims, which should be well separated by the internal pressure of the normal nucleus pulposus, is a likely cause of the osteophytes which form around the periphery of the disc in advanced cases. Apart from the well-known symptoms of brachialgia or brachial neuritis and pain in the neck, these changes are an exceedingly common cause of intractable headaches.

d) Vertebral artery compression or irritation. Hutchinson and Yates drew attention to the effect of narrowing of the vertebral artery upon the cerebral circulation as a whole. In spondylotic spines osteophytes from the Luschka joints project laterally and can interfere markedly with the cerebral circulation.

\section{Uncommon causes of Cervicobrachial neuralgia ${ }^{[1]}$}

1. Pancoast's yndrome

2. Infectious spondylitis

3. Cervical bone metastasis

4. Syringomyelia

5. Neuroma

6. Thoracic outlet syndrome

7. Erosive spondyloarthropathy (CRF patients on hemodialysis)

8. Thalamic tumors

9. Platybasia (Spinal disease of a malformed relationship between the occipital bone and cervical spine. It may be caused by Paget's disease.)

10. Lesions within the intervertebral foramina

11. Cervical rib,

12. Aneurysms of the subclavian artery

13. Pressure in the supraclavicular region

14. Superior sulcus tumors.

15. Heart lesions.

These conditions must be ruled out with appropriate investigations

MRI and cervico brachial neuralgia ${ }^{[2]}$

Recent improvements in magnetic resonance imaging techniques have altered our choices in the exploration of cervicobrachial neuralgia (CBN). The use of high-field machines, new acquisition sequences and paramagnetic contrast media has increased the sensitivity and specificity of the MRI method in the detection of degenerative lesions responsible for CBN. These new techniques are essentially based on gradient-echo sequences can be acquired in two or three dimensions, and each of them has its own advantages and drawbacks, but their performance is sufficient for MRI to be now considered a reliable and non-traumatic exploratory method for CBN. Performed after standard radiography, MRI tends to be the first-choice examination in the preoperative evaluation of cervical radiculopathies and myelopathies.

Mechanisms of sympathetic pain ${ }^{[3]}$

In some chronic pain states, notably causalgia and reflex sympathetic dystrophy, activity in sympathetic efferent neurons can exacerbate the pain and sympathectomies relieve it. These patients are said to have sympathetically maintained pain (SMP). In normal tissue, activity in postganglionic sympathetic efferents does not produce pain, nor is it capable of activating nociceptive sensory neurons. It can, however, induce modest firing in some mechanoreceptors.

SMP is often held to result from a vicious circle of events which include changes in peripheral and central somatosensory processes, and most importantly a positive feedback element in 
the form of sympathetic efferent neurons which, by activating sensory neurons in the periphery, completes the vicious circle. Several specific hypotheses have been advanced as to the primary pathophysiological cause of pain in these patients. Suggestions, largely deriving from observations on animal models, include: ephaptic transmission, adrenergic receptors on sensory neurons, indirect coupling of sympathetic and sensory neurons, sensitization of nociceptive afferents, and, in the central nervous system, sensitization of dorsal horn neurones. All these suggestions have some supporting evidence, but none are able to adequately explain all the disturbances seen in patients with SMP.

\section{Complex Regional Pain Syndrome (CRPS)}

Is a neurologic disorder affecting central and peripheral nervous systems. Complex Regional Pain Syndromes are painful conditions that usually affect the distal part of an upper or lower extremity and are associated with characteristic clinical phenomena (Temperature/colour change. Edema. Trophic skin, hair, nail growth abnormalities, Impaired motor function, Hyperpathia/allodynia, Sudomotor changes.)

There are two types

The terms CRPS Type I and CRPS Type II are meant as descriptors of certain chronic pain syndromes. They do not embody any assumptions about pathophysiology. For the most part the clinical phenomena characteristics of CRPS Type I are the same as seen in CRPS Type II. The central difference between Type I and Type II is that, by definition, Type II occurs following a known peripheral nerve injury, whereas Type I occurs in the absence of any known nerve injury Pain with Sympathetic Activity ${ }^{[4]}$

Pain that can be abolished or greatly reduced by sympathetic blockade (for example, a stellate ganglion block) is called sympathetically maintained pain. Pain that is not affected by sympathetic blockade is called sympathetically independent pain. The pain in some CRPS patients is sympathetically maintained; in others, the pain is sympathetically independent. The relation between CRPS and sympathetically maintained pain can be seen in the following Venn diagram:

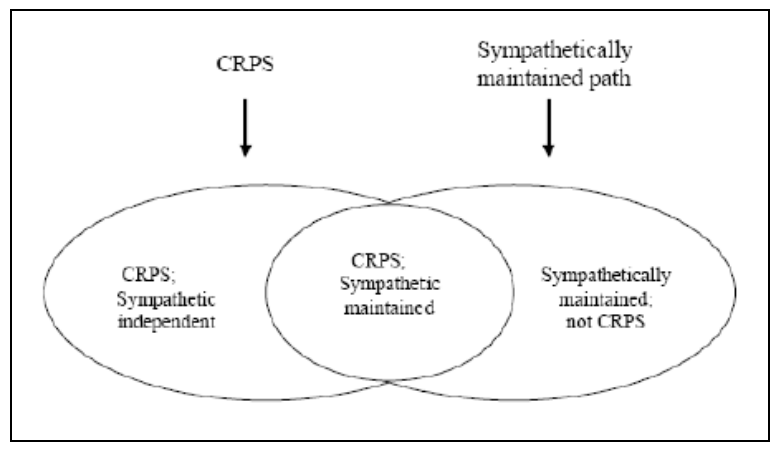

Stellate ganglion block (cervicothoracic sympathetic block) ${ }^{[5,}$ $6,7]$

\section{Indications}

Pain syndromes Complex regional pain syndrome type I and II, Refractory angina, Phantom limb pain, Herpes zoster, Shoulder/hand syndrome, Angina Vascular insufficiency Raynaud's syndrome, Scleroderma, Frostbite, Obliterative vascular disease Vasospasm, Trauma, Emboli

\section{Contraindications}

Coagulopathy, Recent myocardial infarction, Pathological bradycardia, Glaucoma

Anatomy $[8,9,10]$

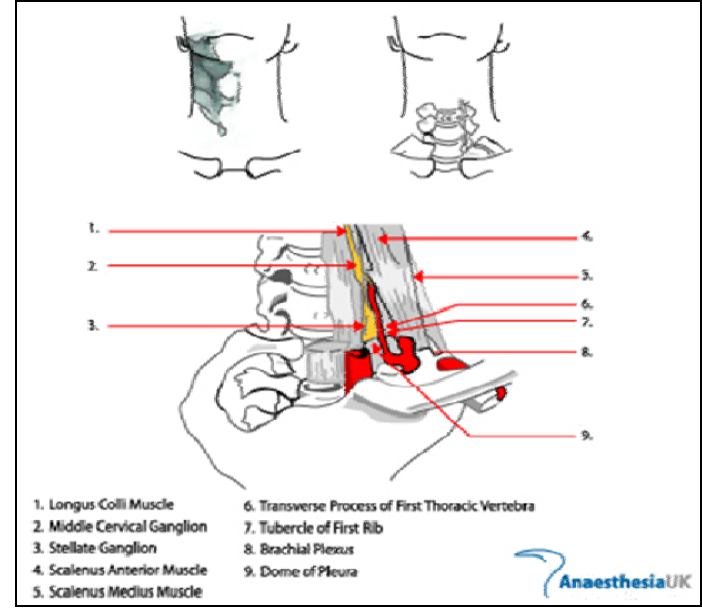

The stellate ganglion refers to the ganglion formed by the fusion of the inferior cervical and the first thoracic ganglion as they meet anterior to the vertebral body of C7. It is present in $80 \%$ of subjects. (In the rest $20 \%$ cases Kuntz nerves exist instead and the block fails).It usually lies on or above the neck of the first rib. The stellate ganglion is a long, flat structure approximately $1 \mathrm{~cm}$ in length. It lies just anterior to the head of the first rib, directly adjacent to the vertebral artery and lateral to the longus colli muscle. It is also closely related to the apex of the lung and the phrenic nerve.

Chassaignac's tubercle

This is the anterior tubercle of the transverse process of the sixth cervical vertebra, which lies lateral to and at a slightly higher level than the posterior tubercle, and against which the carotid artery may be compressed by the finger.

Relations of stellate ganglion

Anterior the structures anterior to the ganglion include the skin and subcutaneous tissue, the sternocleidomastoid and the carotid sheath. The dome of the lung lies anterior and inferior to the ganglion. Medial the prevertebral fascia, vertebral body of C7, oesophagus and thoracic duct lie medially. Posterior Structures posterior to the ganglion include the longus colli muscle, anterior scalene muscle, vertebral artery, brachial plexus sheath and neck of the first rib.

Technique ${ }^{[6,7,11]}$

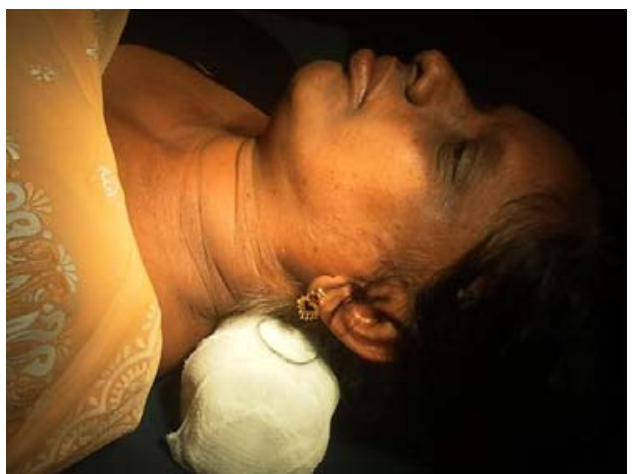

The patient is placed in the supine position with the neck slightly extended, the head rotated slightly to the side opposite the block, and the jaw open. The point of needle puncture is located between

The trachea and the carotid sheath at the level of the cricoid cartilage and Chassaignac's tubercle. Although the ganglion lies at the level of the C7 vertebral body, the needle is inserted at the level of C6 to avoid the piercing the pleura. Cutaneous anaesthesia is obtained with a skin wheal of local anaesthetic. 
Blunting of needle 20 Gauge IV cannula needle is blunted using a sterile straight scissors and this needle is used for stellate block. This needle is kept sterile. The sternocleidomastoid and carotid artery are retracted laterally as the index and middle fingers palpate Chassaignac's tubercle.

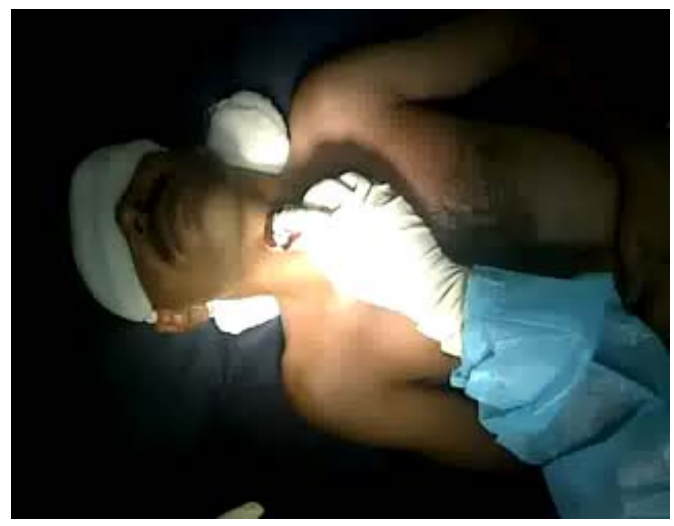

The skin and subcutaneous tissue are pressed firmly onto the tubercle to reduce the distance between the skin surface and bone, and in an attempt to push the dome of the lung out of the path of the needle. When properly performed, this manoeuvre is uncomfortable for the patient. Skin entry is made using a 18 g needle. Then the blunted needle is directed onto the tubercle, and then redirected medially and inferiorly toward the body of C6. After the body is contacted, the needle is withdrawn 1-2 $\mathrm{mm}$. This brings the needle out of the belly of the longus colli muscle, which sits posterior to the ganglion and runs along the anterolateral surface of the cervical vertebral bodies. The needle is then held immobile. A $10 \mathrm{ml}$ control syringe charged with local anaesthetic ( $10 \mathrm{ml} 1 \%$ lignocaine with adrenaline) is attached to the needle and aspiration is performed to rule out intravascular placement. The local anaesthetic (10ml $1 \%$ lignocaine with adrenaline) is injected in divided doses of $3 \mathrm{ml}$ with intermittent aspiration. The patient is placed in the sitting position to facilitate the spread of anaesthesia inferiorly to the stellate ganglion. The onset of Horner's syndrome indicates a successful block.

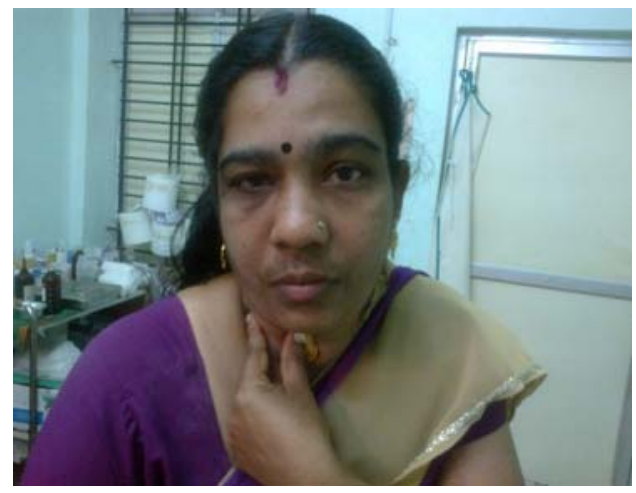

Horner's syndrome is characterized by an interruption of the oculosympathetic nerve pathway somewhere between its origin in the hypothalamus and the eye. The classic clinical findings associated with Horner's syndrome are ptosis, pupillary miosis and facial anhidrosis. Other findings may include apparent enophthalmos, increased amplitude of accommodation, heterochromia of the irides (if it occurs before the age of 2 years), paradoxical contralateral eyelid retraction, and transient decrease in intraocular pressure and changes in tear viscosity.
Complications ${ }^{[6,7]}$

Vascular injury

Neural injury

Pulmonary injury

Oesophageal perforation

Intravascular injection

Epidural block

Intrathecal

Horseness (recurrent laryngeal nerve)

Elevated hemidiaphragm (phrenic nerve)

Infection.

\section{Materials and Methods}

The study was done in Govt. TD Medical College, Alleppey, Kerala, India between a period of 2015 July and 2016 august for Adults with cervicobrachial neuralgia both acute and chronic evaluated clinically and radiologically to rule out malignancies and mechanical severe root compression. Total number of patients included in the study were 35 of which 10 were males and 25 were females [chart-2]. Out of the 35 patients 3 patients were between 20 and 29 years of age while 11 patients were between 30 and 39 years of age, 13 patients were between 40 and 49 years, 5 patients were between 50 and 59 years and 3 patients were between 60 and 69 years [chart1]. Out of the 35patients 2 patients were fishermen, 11 patients were housewives. Others had different occupations

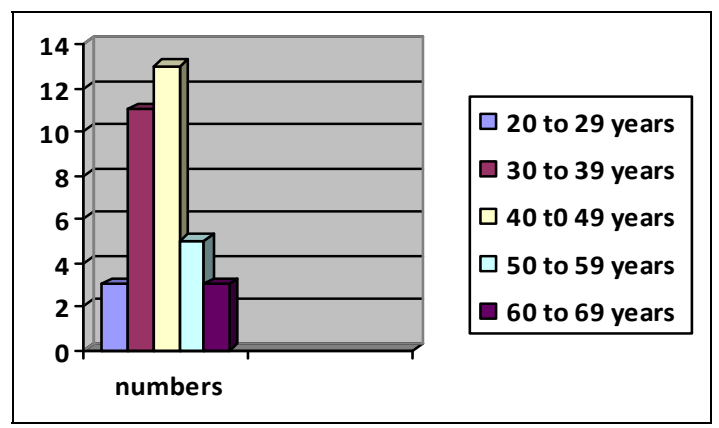

Chart 1: age distribution

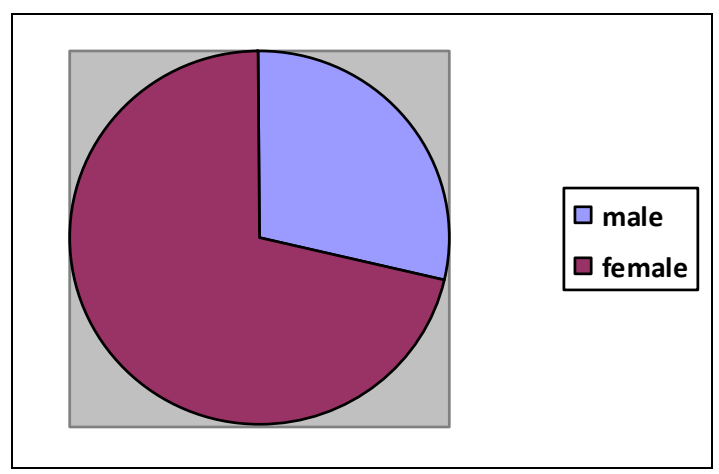

Chart 2: Sex distribution

Three sessions of stellate block is performed in patients at biweekly intervals using $10 \mathrm{ml} 1 \%$ lignocaine with adrenaline. There after feasible lifestyle modifications are advised without interfering personal and professional activities. Isometric neck muscle exercises are advised to be performed two sessions daily.

\section{Inclusion criteria}

Patients with cervicobrachial neuralgia of non-terminal illness of either sex. 
Patients with both acute and chronic symptoms

Patients in the age group of 18-60 years.

Exclusion criteria

Patient On anticoagulants

Bleeding disorders

Recent MI

Bradycardia

Neck extension limited

Well established neurological deficit

Patients with no Horners syndrome developing after first dose of stellate block

Defaulter after $1^{\text {st }}$ session of stellate block.

Sample size- 35 patients in the age group 25 to 60 years were enrolled in the study after getting informed consent.

Study Variables

Onset of Horners syndrome

Pain relief (visual analogue scale)

Side effects if any

\section{Outcome measures}

The final outcome was measured after the 3 biweekly stellate ganglion injection sessions and the patients trained to do the isometric neck muscle exercises. Life style modifications to minimize neck strain are also advised. They were followed up at 1month 3 months 6 months and 1 year. Most of the patients are having cervical spondylosis and strict compliance to regular exercises and life style modifications may not be observed many a times in these group of patients. Final outcome was assessed by VAS score. VAS score 0 good final outcome.VAS1 fair. More than VAS 2 poor results

\section{Data collection methods \&instruments}

For the selection of subjects clinical and Xray examination with base line blood investigations (RBS Hb TC DC ESR BT $\mathrm{CT}$ ). In required cases MRI study to rule out malignancies/ specific root compression due to mechanical cause. In our study half of the patients had underwent MRI examination at the time of starting study

Visual analog scale used to assess the severity of pain, and hence to assess the final outcome

Plan of Data analysis

After collecting data on clinical recovery and complications the following rates were calculated

Pain relief, Resumption of work, Infection rates, Complication rates

Study is compared with similar studies conducted on drug based management of cervicobrachial neuralgia

\section{Ethical considerations}

A) Study was conducted only after getting approval from institutional ethical committee.

B) A written informed consent was taken from all the patients included in the study

C) Stellate ganglion block is a standard procedure practiced in pain management and performed by trained doctors. We are doing the procedure using blunted needle and the described complications are nullified by this step.

\section{Time plan List of activities and time taken}

\begin{tabular}{|c|c|}
\hline Stellate blocks & -At every $\mathbf{2}$ weeks \\
\hline Isometric exercises neck muscles & -after $3^{\text {rd }}$ stellate block \\
\hline Follow up & $\begin{array}{c}1 \text { month } 3 \text { months } 6 \text { months } 1 \\
\text { year }\end{array}$ \\
\hline Final outcome assessment & 1 year \\
\hline
\end{tabular}

\section{Results and discussion}

All petients were followed up for 1 year after 3 sessions of biweekly stellate block at 1 month 3 months 6 months and 1 year intervals. The outcomes were measured using Visual Analogue scale. At I year follow up 32 out of 35 patients had good results (VAS 0). 3 patients had fair results (VAS 1). 17 out of 35 patients had 50 percent relief of pain after the first injection. It is observed that once the severity of pain was reduced by the stellate block the patients could do isometric neck exercises more effectively and thus their VAS score improved further at follow ups. We had no complications.

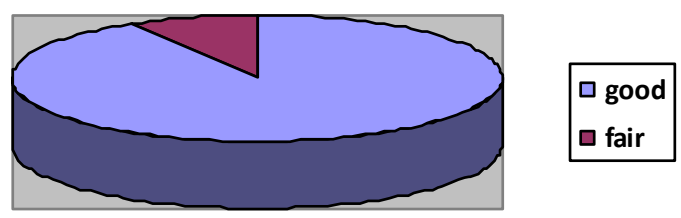

Other methods of management include medications like Gabapentin. According to Moore, RA; Wiffen, PJ; Derry, S; Toelle, T; Rice, AS (27 April 2014). "Gabapentin for chronic neuropathic pain and fibromyalgia in adults." (The Cochrane database of systematic reviews) about $14 \%$ of people have a meaningful benefit. Common adverse effects include dizziness, fatigue, drowsiness, ataxia, peripheral edema and sexual dysfunction in some patients. Serious side effects may include an increased risk of suicide, aggressive behaviour, and drug reaction with eosinophilia and systemic symptoms it is unclear if it is safe during pregnancy or breastfeeding. Restricted use is suggested in impaired renal function.

Amitriptyline is another drug commonly used in the management of similar symptomatology. This drug also has adverse effects like dizziness, headache, weight gain, cognitive effects such as delirium and confusion, mood disturbances such as anxiety and agitation, cardiovascular side effects such as orthostatic hypotension and sinus tachycardia, sexual side effects such as loss of libido and impotence, and sleep disturbances such as drowsiness, insomnia and nightmares

Stellate Ganglion block is a very cost effective method of symptomatic management of cervicobrachial neuralgia. It gives immediate pain relief in severe cases and predictable improvement in the character of pain. With the blunt needle technique this procedure carries very minimal risk. This procedure can be performed as OP procedure. Adverse effects of medications like Tricyclic ante-depressants can be avoided

\section{References}

1. Cheikhrouhou Abdelmoula L, Daoud L, Ben Hadj Yahia C, Tekaya R, Chaabouni L et al. Source Service de rheumatologie Tunis Med. [Uncommon cervicobrachial neuralgia: about 17 cases]., Hospital Charles Nicolle, Tunis, Tunisie 2011; 89(7):598-603.

2. Magnetic Resonance Imaging in the Diagnosis of Disc Degeneration: Correlation with Discography. Schneiderman, Gary Md; Flannigan, Bonnie Md; Kingston, Scott Md; Thomas, James Md; Dillin, William H. Md; Watkins, Robert G. Md Spine journal. 1987; 12(3):197-308.

3. Stanton-Hicks M, Janig W, Hassenbusch S, Haddox JD, Boas R, Wilson P. Reflex sympathetic dystrophy: changing concepts and taxonomy. Pain. 1995; 63(1):12733.

4. Janig W, Baron R. Complex regional pain syndrome is a disease of the central nervous system. Clin Auton Res. 2002; 12(3):150-64. 
5. Atlas of Image-Guided Intervention in Regional Anesthesia and Pain Medicine James P. Rathmell Lippincott Williams \& Wilkins, 2011.

6. Cousins and Bridenbaugh's Neural Blockade in Clinical Anesthesia and Pain Medicine Michael J. Cousins Lippincott Williams \& Wilkins, 29-Mar-2012.

7. Current Therapy in Pain Howard S. Smith Elsevier Health Sciences, 2009

8. The Organization of the Stellate Ganglion: A Study of Synaptic Architecture and Amacrine Neurons in OctopusEdwin Moreland Monsell Duke University, 1977.

9. Clinical Anatomy by Regions Richard S. Snell Lippincott Williams \& Wilkins, 2008.

10. Concise Anatomy for Anaesthesia Andreas G. Erdmann Cambridge University Press, 2001

11. Malmqvist EL, Bengtsson M, Sorensen J. Efficacy of stellate ganglion block: A clinical study with bupivacaine. Regional Anesthesia 1992; 17:340-7.

12. Fukusaki M, Matsumoto M, Yamguchi K, Nakamura $H$, Sumikawa K. The role of nerve blocks to deal with pain associated with cervical radiculopathy. Pain clinic 1995; 8:219-225

13. Wewers ME, Lowe NK. A critical review of visual analogue scales in the measurement of clinical phenomena. Research in Nursing and Health 1990; 13:227-236.

14. Annexures -Questionnaire/consent form 